\title{
Discrepancy in the preferences of place of death between terminally ill cancer patients and their primary family caregivers in Taiwan
}

\author{
Siew Tzuh Tang ${ }^{\mathrm{a}, *}$, Tsang-Wu Liu ${ }^{\mathrm{b}}$, Mei-Shu Lai ${ }^{\mathrm{c}}$, Ruth McCorkle ${ }^{\mathrm{d}}$ \\ ${ }^{a}$ National Yang-Ming University, School of Nursing, \# 155, Sec. 2, Li-Nong Street, Taipei, Taiwan, R. O.C \\ ${ }^{\mathrm{b}}$ Taiwan Cooperative Oncology Group, 3F, 109, Min-Chuan East Road, Sec.6, Taipei 114, Taiwan, R. O.C. \\ ${ }^{\mathrm{c}}$ Division of Preventive Medicine, The Institute of Public Health, College of Public Health, National Taiwan University, \\ Rm. 211, No. 19, Hsuchow Rd, Taipei, Taiwan, R. O.C \\ ${ }^{\mathrm{d}}$ Center for Excellence in Chronic Illness Care, School of Nursing, Yale University, P. O. Box 9740, New Haven, CT, 06536-0740
}

Available online 7 April 2005

\begin{abstract}
There is a worldwide common preference for dying at home. However, death at home does not come without significant challenges and potential consequences for families. Given the interactive nature of decisions regarding the place of death, the family's perspective is important and needs to be investigated. The purposes of this study were to compare (1) Taiwanese terminally ill cancer patients' and their family caregivers' preferences for the patient's place of death; and (2) important factors that are considered in choosing the preferred place of death from both points of view.

A total of 617 dyads of terminally ill cancer patients and their family caregivers were surveyed. The majority of both terminally ill cancer patients and their family caregivers preferred to die at home $(61.0 \%$ and $56.9 \%$, respectively). A higher proportion of the family caregivers indicated a preference for hospital death for the patients. There was a moderate association between the two respondents in the preferences of place of death.

Results underscore discrepancies between patients and their families in the importance given to cultural concerns, quality of health care, worries of being a burden to others, lack of availability of families, relationships with health care providers, and being surrounded by the home environment.

Effective interventions need to be developed which can lighten the caregiving burden and help families retain dying patients at home, avoid unnecessary re-hospitalizations and unfavorable hospital deaths, and improve accordance with the patient's wishes.
\end{abstract}

(C) 2005 Elsevier Ltd. All rights reserved.

Keywords: End-of-life care; Terminally ill cancer patients; Place of death; Taiwan

\section{Introduction}

\footnotetext{
*Corresponding author. Tel.: +886228267033; fax: +886228203080 .

E-mail addresses: sttang@ym.edu.tw (S.T. Tang), walter@nhri.org.tw (T.-W. Liu), mslai@episer.cph.ntu.edu.tw (M.-S. Lai), ruth.mccorkle@yale.edu (R. McCorkle).
}

The basic tenets of end-of-life care have been suggested as philosophically rooted in recognition of the inherent dignity of individuals (Latimer, 1991; Pullman, 1996) and one of the main goals of end-oflife care is to help patients die with dignity (Chochinov, 
2002; Pullman, 1996). To maintain dignity, terminally ill cancer patients must have a sense of control or autonomy over their dying circumstances and must be able to make their own end-of-life care decisions (Chochinov, Hack, McClement, Kristjanson, \& Harlos, 2002; Duda, 1982). One specific task that patients must make is to decide where they prefer to spend their final days of life and to die (Patrick, Engelberg, \& Curtis, 2001). The setting affects the philosophy of care as well as the type and intensity of services that can be delivered to the dying; therefore, setting has a tangible, direct, and immediate impact on the quality of life that a patient experiences at the end of life or even the quality of death itself (Mezey, Dubler, Mitty, \& Brody, 2002). Patrick et al. (2001) specifically defined one of the domains of quality of dying and death as dying at the place of one's choice.

Reviews from empirical studies (Higginson \& SenGupta, 2000; Tang \& McCorkle, 2001) show that the great majority (approximately two-thirds) of cancer patients, when asked about their preferred place of death, wish to spend their final days of life and die at home. Home is a place where people may feel safety and a sense of belonging (Gott, Seymour, Bellamy, Clark, \& Ahmedzai, 2004). When dying at home, terminally ill cancer patients also may have a greater chance to control their environment, more autonomy and privacy, and a sense of normality (Tang, 2003). Particularly, for Chinese people, dying at home has a special cultural meaning (Tang, 2000). When death occurs at home, the spirit of the dead can reunite with the forebears, thus, "the fallen leaves can return to their roots". When one dies at home, the spirit of the dead has a place to rest and the dead will not be a 'koo'un' $i a^{\prime} k u i$ '(spirit wanderer), a solitary soul with no one to depend on. Furthermore, a death at home is believed to be crucial to the well-being of the living descendants. Chinese believe that the dead family member will intercede with a supernatural power on behalf of the descendants for special blessings. In addition, the Chinese recognize that death is the utmost adversity in human life. The deceased is the victim of the family's disaster. When he dies, he takes the misfortune of earthly life and leaves good fortune to the family. However, if an individual has died outside the home, the person lacks the domain through which to shelter or bless his descendants.

Despite the worldwide unanimous strong preference for dying at home, the reality is that, when the time comes, few cancer patients achieve their preferences in regard to the place of death (Grande, Addington-Hall, \& Todd, 1998; Hunt, Fazekas, Luke, \& Roder, 2001; Gatrell et al., 2003), as research has shown for many decades. Mor and Hiris (1983) recognized the determinants of place of death as a function of a complex interplay of personal and cultural values, support network characteristics, physical and medical factors, and various health care systems forces. From over 50 studies investigating the determinants of place of death for terminally ill cancer patients, few definite conclusions can be made (Higginson \& Sen-Gupta, 2000; Tang \& McCorkle, 2001). The availability of health care resources (i.e., acute care hospital beds, hospice care, and physician supplies) has been documented as the most powerful determinant of place of death (Gallo, Baker, \& Bradley, 2001; Mor \& Hiris, 1983; Pritchard et al., 1998; Virning, McBean, King, \& Dholakia, 2002) followed by the preference for dying at home (Cantwell et al., 2000; Hinton, 1994a; Karlsen \& Addington-Hall, 1998; McWhinney, Bass, \& Orr, 1995). Other frequently observed factors are the availability, ability, and attitudes of family caregivers (Cantwell et al., 2000; Clifford, Jolley, \& Giles, 1991; Fukui, Fukui, \& Kawagoe, 2004; Mor \& Hiris, 1983; Visser et al., 2004). Specifically, Cantwell et al. (2000) reported that the main predictor of home death for terminally ill cancer patients under palliative home care was the agreement between the patient and the family caregiver. If there is a desire for a home death by both, the likelihood of dying at home is 8.38 times higher than when there is lack of agreement.

One of society's great assets is the many family members who provide care to ill or dying relatives. This nurturing and caring role of the family is even more prominent in Taiwan, based on the filial duty which is strongly emphasized in Asian cultures. Chinese (including Taiwanese) follow the doctrine that children should take care of parents when they are aged, sick, or even dying in return of parents' efforts in bringing them up. However, death at home does not come without significant challenges and potential consequences for families. While many caregivers value their caregiving role, the achievement of the patient's preference of dying at home involves their own emotional and physical sacrifice as well as profound economic difficulties for an undetermined length of time (Christakis \& Iwashyna, 2003; Donelan et al., 2002; Emanuel, Fairclough, Slutsman, \& Emanuel, 2000; Schulz et al., 2001). Given the interactive nature of decisions regarding the place of death, the family's perspective is an important force that needs to be further investigated. Patients and family members may or may not be in rhythm as they deal with the anticipated death or in their preferences of the place of death. The purposes of this study were to: (1) compare Taiwanese terminally ill cancer patients' and their primary family caregivers' preferences for the patient's place of death; and (2) explore and compare the factors that are considered as important in choosing the preferred place of death from both points of view. Currently, Chinese (including Taiwanese) represent nearly a quarter of the world's population, and encountering them becomes common in clinicians' everyday practice. Findings from this study may have 
implications for end-of-life care in other parts of the world.

\section{Method}

A survey was conducted among 21 hospitals which provide the great majority of care for cancer patients in Taiwan from February 2003 to May 2004. This report is part of larger research funded by the Department of Health in Taiwan. The human subject research review committees of study hospitals granted approval of this study and permission to contact patients was obtained from the primary physicians of study subjects. Subjects were recruited by a convenience sampling strategy, inviting all terminally ill cancer patients in the inpatient wards at the time who met the following eligibility criteria: patients: (1) who had a disease at a terminal stage which continued to progress with distant metastases and was unresponsive to current curative cancer treatments; (2) who were cognitively competent; (3) who could communicate with data collectors; and (4) whose designated family caregivers agreed to participate in the survey. The family caregiver was defined as the person most involved in the patient's care and health care decision making. Primary physicians in each study site were given a detailed explanation regarding the purposes of the study and were asked to identify patients without judging the emotional readiness for the patients to talk about their preferences for place of death. After verifying the eligibility of patients, data collectors invited potential patients to participate in this study without asking permission from their family to avoid the exclusion of patients due to family caregivers' opinions about the suitability of patients' participation. Written informed consent was obtained from all participants, including patients and their family caregivers.

\section{Data collection process}

Data were gathered from patients' medical records and from interviews with patients and their identified primary family caregivers. Semi-structured interviews were used to explore preferences for place of death for the patient and the reasons for the decisions that were made. Considering the sensitivity of the topic, in order not to emotionally disturb the participants, patients were asked: "Some people prefer to die at home, whereas other people prefer to die in a hospital, a hospice, or a nursing home. One day, if you need to consider this issue, where would you prefer to be? Why do you choose (home, hospital, inpatient hospice, or others) as the preferred place?'. The same questions were presented to family caregivers but asking about their preferences for the patient's place of death and the reasons for that decision. Consenting patients and family caregivers were interviewed separately to ensure independent responses. Participants were invited to discuss their preferences and the reasons for them in detail. Responses to the research questions were coded based on the data collectors' judgments. Initial categorization of reasons for choosing a preferred place of death was derived from the literature (Gott et al., 2004; Tang, 2003; Thomas, Morris, \& Clark, 2004).

To standardize presentation of questions, interviewers were trained in conducting the interviews through smallgroup didactic sessions and individually coached by the principal investigator and two senior project managers. The initial interviews of each data collector were conducted under supervision and participant responses were coded and interpreted by both the data collector and the supervisor individually. Any discrepancies of coded responses were discussed and resolved, and consensus was achieved before each data collector conducted interviews independently.

\section{Statistical analysis}

Descriptive statistics were compiled of the characteristics of the subjects and to present frequencies of preferences of place of death and the reasons for choice. The extent of differences in preferences between patients and their primary family caregivers was analyzed using chi-square for multinominal data and the McNemar test to examine differences in the proportions of binominal responses for paired data, respectively. A $p$ value less than 0.004 was judged as significant based on a Bonferron-corrected $p$ value $(0.05 / 12=0.004)$ to adjust for the potential Type I errors that may be derived from multiple comparisons (Kleinbaum, Kupper, Muller \& Nizam, 1998). In addition to percentage of agreements, kappa coefficients were computed to correct or adjust for the amount of agreement that can be expected to occur by chance alone (Feinstein \& Cicchetti, 1990). Criteria proposed by Landis and Koch (1977) for kappa as a measure of the strength of agreement were followed: $\leqslant 0.20$, poor; 0.21-0.40, fair; 0.41-0.60, moderate; $0.61-0.80$, substantial; $0.81-1.00$, almost perfect.

\section{Results}

A total of 617 dyads of terminally ill cancer patients and their primary family caregivers were recruited from 907 eligible pairs of subjects $(68.0 \%$ participation rate). Age was the only significant difference in socialdemographic and medical characteristics between the patient participants and non-participants. Patients who participated in this study were significantly younger than the non-participants by a mean age of 3 years $(p=0.0003)$. Except for gender, there were no differences in age and relationships with patients between the 
family participants and non-participants. More male family caregivers participated in this study $(p=0.006)$. Primary reasons for declining to participate were fragile physical condition $(n=201 ; 69.3 \%)$ for the patients and lack of interest $(n=161 ; 55.5 \%)$ for the family caregivers.

Slightly greater than half of the patients $(58.5 \%)$ were male. The median age of patients was 62 years (range: 22-89 years). The majority of patients were married $(83.9 \%)$, lived with their spouse $(77.8 \%)$ and children $(75.0 \%)$, and had an educational level equal to or less than high school $(67.8 \%)$. The most common diagnoses of the patients were lung cancer $(30.0 \%)$, colon-rectal cancer $(9.6 \%)$, hematological malignancies $(9.4 \%)$, hepatoma $(7.8 \%)$, and head and neck cancer $(7.4 \%)$. The median length of time since diagnosis was 9 months (range: 1-288 months). At the time of the interview, a quarter of the patients had disease metastasized to bone, lung, liver, or brain and only $6.9 \%$ had a do-notresuscitate order in place. For patients whose information on the date of death was available, the interviews were conducted an average 33.8 days (standard deviation: 41.9 days, range: 1-242 days, median: 18.0 days) before death.

Approximately two-thirds $(63.4 \%)$ of the primary family caregivers were female and half of them $(51.9 \%)$ were the patient's spouse and the other $31.8 \%$ the patient's children. The median age of family caregivers was 48 years (range: 17-85 years). Over four-fifths $(82.8 \%)$ of the family caregivers were married and $78.4 \%$ of them lived with the patient. Over four-fifths $(87.2 \%)$ of the family caregivers had contacts with the patients daily. Approximately two-thirds (61.4\%) of the caregivers believed they knew the patient's thoughts and feelings adequately or very well.

\section{Preferences of place of death}

A majority of both patients and their caregivers preferred to die at home $(61.0 \%$ and $56.9 \%$, respectively) (Table 1). Hospital was the second preference identified by both. Fewer than 5\% from both groups identified inpatient hospices as their preferred place of death. Approximately $9 \%$ and $8 \%$ of the patients and their family caregivers, respectively, indicated no preference for place of death. Chi-square statistic showed a significant difference in the preferences of place of death between the patients and their family caregivers. Overall, there was $74.1 \%$ agreement in the preferences for place of death. The kappa coefficient of agreement was 0.55 (95\% CI: 0.49-0.61), indicating a moderate association between the two respondents in their preferences.

Table 1

Preferred place of death between terminally ill cancer patients and their families

\begin{tabular}{|c|c|c|c|c|c|}
\hline \multirow[t]{2}{*}{ Variables } & Patients & Families & $\% *$ & $\chi^{2} \mathrm{McNemar}$ & Kappa $(95 \%$ CI $)$ \\
\hline & \multicolumn{5}{|l|}{$(\%)$} \\
\hline \multicolumn{6}{|l|}{ Preferred place of death $(N=559)$} \\
\hline Home & 61.0 & 56.9 & 74.1 & 534.4 & $0.55(0.49,0.61)$ \\
\hline Hospital & 24.2 & 28.8 & & $(\mathrm{df}=16)$ & \\
\hline Inpatient hospice & 2.0 & 3.2 & & $P<0.0001$ & \\
\hline No preference & 9.3 & 8.1 & & & \\
\hline Others & 3.6 & 3.0 & & & \\
\hline \multicolumn{6}{|c|}{ Reasons for choosing the preferred place of death $(N=556)$} \\
\hline Cultural concerns & 34.0 & 21.2 & 75.4 & $36.8 * *$ & $0.40(0.32,0.48)$ \\
\hline Being with families & 31.3 & 29.7 & 77.9 & 0.7 & $0.48(0.40,0.56)$ \\
\hline Familiar and comfortable home environment & 21.9 & 16.0 & 77.2 & $8.6^{\dagger \dagger}$ & $0.26(0.17,0.36)$ \\
\hline Having greater autonomy & 12.2 & 13.9 & 86.2 & 1.1 & $0.39(0.28,0.50)$ \\
\hline Enjoying "normal" life & 12.0 & 11.3 & 88.9 & 0.3 & $0.46(0.35,0.57)$ \\
\hline Difficulty in managing the deceased's body & 17.8 & 16.9 & 85.8 & 0.3 & $0.51(0.41,0.60)$ \\
\hline Quality of health care & 13.5 & 21.6 & 78.6 & $17.0^{* *}$ & $0.27(0.17,0.37)$ \\
\hline Burden to others & 9.9 & 4.5 & 89.6 & $15.5^{* *}$ & $0.23(0.10,0.36)$ \\
\hline Lack of ability of families & 5.9 & 9.2 & 90.3 & 6.0 & $0.31(0.17,0.44)$ \\
\hline Lack of availability of families & 4.5 & 8.6 & 92.6 & $12.9^{* * *}$ & $0.40(0.26,0.55)$ \\
\hline Relationships with health care providers & 1.6 & 5.2 & 93.9 & $11.8^{\dagger}$ & $0.08^{\ddagger}(-0.05,0.22)$ \\
\hline Financial concern & 3.4 & 2.9 & 96.6 & 0.5 & $0.44(0.23,0.65)$ \\
\hline
\end{tabular}

$* \%$ of agreement; Except for ${ }^{*}$, all kappa values are significant at $p<0.0001$ level; ${ }^{* *} p<0.0001 ; * * * p=0.0003 ;{ }^{\dagger} p=0.0006$; ${ }^{\dagger \dagger} p=0.003$. 


\section{Reasons for choosing a specific setting as the preferred place of death}

Multiple reasons were provided by the respondents for deciding their preferences (Table 1), including cultural concerns, quality of life, availability and ability of family caregivers, quality of health care, worries of being a burden to others, and concerns about difficulty in managing the decease's body. Cultural concerns were identified by one-third of the patients. Considerations of quality of life also motivated the patients to choose home as their preferred place of death. They mentioned being with their families, being surrounded by familiar and comfortable home environment, having greater autonomy, and enjoying more "normal" life as the principal reasons why they preferred spending the rest of their life at home and dying there. The difficulty of managing the deceased's body was recognized as an important issue for approximately one-fifth of the patients. The majority of people in Taiwan live in a small apartment in buildings with three to five floors without elevators; therefore it is difficult to manage the deceased's body. Concerns about quality of health care and not being a burden to others were reported by $13.5 \%$ and $9.9 \%$ of the patients, respectively. Finally, approximately $5 \%$ of the patients identified the lack of availability or ability of family members to take care of them at home as the reasons that influence their decisions.

Family caregivers indicated similar concerns as the patients but with different priorities (Table 1). Family caregivers gave higher priorities to the considerations of quality of health care and the availability and ability of family caregivers. Results from the McNemar tests showed that when making the decision about the preferred place of death, there were significant differences in considering cultural concerns, quality of health care, worries of being a burden to others (the first three at $p<0.0001$ level), lack of availability of families $(p=0.0003)$, relationships with health care providers $(p=0.0006)$, and being surrounded by a familiar and comfortable home environment $(p=0.003)$. Overall agreement ranged from $75.5 \%$ to $96.6 \%$. Kappa values indicated: (1) moderate agreements on concerns about difficulty in managing the deceased's body $(0.51,95 \%$ CI: $0.41-0.60)$ and financial issues $(0.44,95 \% \mathrm{CI}$ : $0.23-0.65)$; (2) fair to moderate agreements on considerations of quality of life (range from 0.26 to 0.48 ); (3) fair agreements on cultural concerns $(0.40,95 \% \mathrm{CI}$ : $0.32-0.48)$, lack of availability or ability of families to take care of patients at home (kappa $(95 \% \mathrm{CI})=0.40$ $(0.26-0.55)$ and $0.31(0.17-0.44)$, respectively), quality of health care $(0.27,95 \% \mathrm{CI}: 0.17-0.37)$, and worries about being a burden to others $(0.23,95 \% \mathrm{CI}$ : $0.10-0.36)$; and (4) poor agreement on concerns of relationships with health care providers $(0.08,95 \% \mathrm{CI}:-0.05-0.22)$.

\section{Discussion}

This study confirmed the findings in the literature that the majority of terminally ill cancer patients and their primary family caregivers preferred death at home (Higginson \& Sen-Gupta, 2000; Tang \& McCorkle, 2001). Considering the limited availability (currently, 341 hospice beds in total for the whole country) and the fact that inpatient hospice units are not widely accepted, due to their association with the negative images of "death wards" in Taiwan, inpatient hospice units were infrequently identified as the preferred place of death by both sets of participants. Nevertheless, results from this study show great discrepancies between the two groups of respondents in the preferences of place of death and in the factors that were considered as important in making the decision about the preferred place of death. Although the overall agreement on the issues was high, kappa statistics indicated that agreement on all issues was at the best moderate. This highlights that this group of terminally ill cancer patients and their primary family caregivers were not in rhythm in their preferences and concerns for place of death. A higher proportion of the family caregivers indicated a preference for hospital death for the patients, but a higher proportion of the patients preferred to die at home. This discrepancy in attitudes has implications for end-of-life care. Recent literature suggests a transition from respect for patient autonomy, to respect for the patient as a person situated in a social and cultural context (Ballard-Reisch \& Letner, 2003). End-of-life care decisions do not happen as discrete and isolated events; rather, they are family events (Dudgeon \& Kristjanson, 1995). Wishing to die at home and having that wish come true requires not only advance planning, but also is heavily dependent on family caregivers being available, willing, healthy, and capable to support patients in the home (McWhinney et al., 1995; Visser et al., 2004). More intensive research is needed to investigate the challenges and difficulties for families in taking care of dying patients at home.

Apart from the unique cultural concerns and concerns for the difficulty in managing the deceased's body for Taiwanese terminally ill cancer patients, considerations in choosing the preferred place of death found in this study were identical to those documented from the research conducted in the United States and in England (Gott et al., 2004; Tang, 2003; Thomas et al., 2004). When terminally ill cancer patients and their primary family caregivers made the decision regarding the preferred place of death, they not only weighed personal values (family contacts, autonomy, privacy, a sense of normality, and quality of life) but also took into consideration the family caregiving burden, health care needs, and availability of health care resources to meet the patients' needs at the end of life. 
Although family caregivers appreciate the quality of life that the patient can enjoy while dying at home, they were realistic about the support systems required for achieving the patient's preference for dying at home. Advanced cancer imposes numerous adverse symptoms on the patients and great burden of sustained personal and skillful care for family caregivers at the end of life (Emanuel et al., 1999). Without adequate professional and community help, few family caregivers can meet the physical and emotional demands of caring for dying patients at home on their own. Our society should recognize and support the substantial amount of time and efforts family members devote to caring for terminally ill patients. Effective interventions need to be developed to provide care tailored towards empowering families to take care of terminally ill cancer patients at home and reducing their unmet needs. Such interventions could lighten their caregiving burden and in turn help families retain their dying family members at home longer, avoid unnecessary rehospitalizations and unfavorable hospital deaths, and improve the extent of deaths in a place that is in accord with the patient's wish. With these efforts, a high quality of life at the end of life, and a good death, may be achieved.

The strengths of this study include the large sample size and the first direct comparison of preferences of place of death between terminally ill cancer patients and their primary family caregivers. However, representativeness may be compromised by the convenience sampling scheme of this study. Nevertheless, the distribution of gender, age, and disease categories of the patient subjects in this study are similar to the statistics of all cancer patients who died in Taiwan in 2003 (Department of Health, 2004). The generalizability of the findings from this study may not be applied to terminally ill cancer patients receiving care other than at the 21 study sites and residing in other geographic areas. In addition, preferences may change in response to worsening physical symptoms, diminished family resources, available health care system factors and other variables (Hinton, 1994b). Cross-sectional assessments may not be able to capture the dynamic decision-making process surrounding preferences of place of death and the factors that may change as patients approach death. Despite these limitations, results from this study are informative. It is shown that there is a great discrepancy between the preferences of place of death between these patients and their family caregivers. In view of the cultural meanings and quality of life of dying at home, effective interventions need to be developed to empower family caregivers to meet the challenges inherent in providing end-of-life care at home. Thus, a "good death" as recommended by the Institute of Medicine (1997) may be facilitated.

\section{References}

Ballard-Reisch, D. S., \& Letner, J. A. (2003). Centering families in cancer communication research: Acknowledging the impact of support, culture and process on client/provider communication in cancer management. Patient Education and Counseling, 50, 61-66.

Cantwell, P., Turco, S., Brenneis, C., Hanson, J., Neumann, C. M., \& Bruera, E. (2000). Predictors of home death in palliative care cancer patients. Journal of Palliative Care, 16, 23-28.

Chochinov, H. M. (2002). Dignity-conserving care-a new model for palliative care: Helping the patient feel valued. Journal of American Medical Association, 287, 2253-2260.

Chochinov, H. M., Hack, T., McClement, S., Kristjanson, L., \& Harlos, M. (2002). Dignity in the terminally ill: A developing empirical model. Social Science \& Medicine, 54, 433-443.

Christakis, N. A., \& Iwashyna, T. J. (2003). The health impact of health care on families: A matched cohort study of hospice use by decedents and mortality outcomes in surviving, widowed spouses. Social Science \& Medicine, $57,465-475$.

Clifford, C. A., Jolley, D. J., \& Giles, G. G. (1991). Where people die in Victoria. The Medical Journal of Australia, $155,446-456$.

Department of Health. Causes of cancer deaths. (2004). http://www.doh.gov.tw/statistic/data/死因摘要/92年/92.htm.; Accessed on July 14, 2004.

Donelan, K., Hill, C. A., Hoffman, C., Scoles, K., Feldman, P. H., Levine, C., \& Gould, D. (2002). Challenged to care: Informal caregivers in a changing health system. Health Affairs, 21(4), 222-231.

Duda, D. (1982). A guide to dying at home. New Mexico: John Mair Publishing Inc pp. 58-71.

Dudgeon, D. J., \& Kristjanson, L. (1995). Home versus hospital death: Assessment of preferences and clinical challenges. Canadian Medical Association Journal, 152, 337-340.

Emanuel, E. J., Fairclough, D. L., Slutsman, J., Alpert, H., Baldwin, D., \& Emanuel, L. L. (1999). Assistance from family members, friends, paid care givers and volunteers in the care of terminally ill patients. The New England Journal of Medicine, 341(13), 956-963.

Emanuel, E. J., Fairclough, D. L., Slutsman, J., \& Emanuel, L. L. (2000). Understanding economic and other burdens of terminal illness: The experience of patients and their caregivers. Annual Internal Medicine, 132, 451-459.

Feinstein, A. R., \& Cicchetti, D. V. (1990). High agreement but low kappa: I. The problems of two paradoxes. Journal of Clinical Epidemiology, 43, 543-549.

Fukui, S., Fukui, N., \& Kawagoe, H. (2004). Predictors of place of death for Japanese patients with advanced-stage malignant disease in home care settings: A nationwide survey. Cancer, 101, 421-429.

Gallo, W. T., Baker, M. J., \& Bradley, E. H. (2001). Factors associated with home versus institutional death among cancer patients in Connecticut. Journal of the American Geriatric Society, 49, 771-777.

Gatrell, A. C., Harman, J. C., Francis, B. J., Thomas, C., Morris, S. M., \& McIllmurray, M. (2003). Place of death: 
An analysis of cancer death in part of North West England. Journal of Public Health Medicine, 25, 53-58.

Gott, M., Seymour, J., Bellamy, G., Clark, D., \& Ahmedzai, S. (2004). Older people's views about home as a place of care at the end of life. Palliative Medicine, 18, 460-467.

Grande, G. E., Addington-Hall, J. M., \& Todd, C. J. (1998). Place of death and access to home care services: Are certain patient groups at a disadvantage? Social Science \& Medicine, 47, 565-579.

Higginson, I. J., \& Sen-Gupta, G. J. A. (2000). Place of care in advanced cancer: A qualitative systematic literature review of patient preferences. Journal of Palliative Medicine, 3, 287-300.

Hinton, J. (1994a). Which patients with terminal cancer are admitted from home care? Palliative Medicine, 8, 197-210.

Hinton, J. (1994b). Can home care maintain an acceptable quality of life for patients with terminal cancer and their relatives? Palliative Medicine, 8, 183-196.

Hunt, R. W., Fazekas, B. S., Luke, C. G., \& Roder, D. M. (2001). Where patients with cancer die in South Australia, 1990-1999: A population-based review. The Medical Journal of Australia, 175, 526-529.

Institute of Medicine. (1997). In M. J. Field, \& C. Cassell (Eds.), Approach death: Improving care at the end of life. Washington, DC: National Academy Press.

Karlsen, S., \& Addington-Hall, J. (1998). How do cancer patients who die at home differ from those who die elsewhere? Palliative Medicine, 12, 279-286.

Kleinbaum, D. G., Kupper, L. L., Muller, K. E., \& Nizam, A. (1998). Applied regression analysis and other multivariate methods. (3rd ed., pp. 29-30, 400-403). Pacific Grove, CA: Brooks/Cole Publishing Company.

Landis, J. R., \& Koch, G. G. (1977). The measurement of observer agreement for categorical data. Biometrics, 33, 159-174.

Latimer, E. (1991). Caring for seriously ill and dying patients: The philosophy and ethics. Canadian Medical Association Journal, 144, 859-864.

McWhinney, I. R., Bass, M. J., \& Orr, V. (1995). Factors associated with location of death (home or hospital) of patients referred to a palliative care team. Canadian Medical Association Journal, 152, 361-367.
Mezey, M., Dubler, N. N., Mitty, E., \& Brody, A. A. (2002). What impact do setting and transitions have on the quality of life at the end of life and the quality of the dying process? Gerontologist, 42(Suppl. III), 54-67.

Mor, V., \& Hiris, J. (1983). Determinants of site of death among hospice cancer patients. Journal of Health and Social Behavior, 24, 375-384.

Patrick, D. L., Engelberg, R. A., \& Curtis, J. R. (2001). Evaluating the quality of dying and death. Journal of Pain and Symptom Management, 22, 717-726.

Pritchard, R. S., Fisher, E. S., Teno, J. M., Sharp, S. M., Reding, D. J., Knaus, W. A., et al. (1998). Influence of patient preferences and local health system characteristics on the place of death. Journal of American Geriatrics Society, 46, 1242-1250.

Pullman, D. (1996). Dying with dignity and the death of dignity. Health Law Journal, 4, 197-219.

Schulz, R., Beach, S. R., Lind, B., Martire, L. M., Zdaniuk, B., Hirsch, C., et al. (2001). Involvement in caregiving and adjustment to death of a spouse: Findings from the Caregiver Health Effects Study. Journal of American Medical Association, 285, 3123-3129.

Tang, S. T. (2000). Meanings of dying at home for Chinese patients in Taiwan with terminal cancer: A literature review. Cancer Nursing, 23, 367-370.

Tang, S. T. (2003). When death is imminent, where terminally ill cancer patients prefer to die and why. Cancer Nursing, 26, 245-251.

Tang, S. T., \& McCorkle, R. (2001). Determinants of place of death for terminal cancer patients. Cancer Investigation, 19, 165-180.

Thomas, C., Morris, S. M., \& Clark, D. (2004). Place of death: Preferences among cancer patients and their carers. Social Science \& Medicine, 58, 2431-2444.

Virning, B. A., McBean, M., King, S., \& Dholakia, R. (2002). Hospice use before death: Variability across cancer diagnoses. Medical Care, 40, 73-78.

Visser, G., Klinkenberg, M., van Groenou, M. I. B., Wilems, D. L., Knipscheer, C. P. M., \& Deeg, D. J. H. (2004). The end of life: Informal care for dying older people and its relationship to place of death. Palliative Medicine, 18, 468-477. 